\title{
A PARTIAL ORDER ON ×2-INVARIANT MEASURES
}

\author{
OLIVER JENKINSON
}

Dedicated to the memory of Bill Parry

\begin{abstract}
We introduce a partial order on the set of $\times 2$-invariant probability measures, describing relative dispersion, and show that the minimal elements for this order are the Sturmian measures of Morse \& Hedlund.
\end{abstract}

\section{Introduction}

Iteration of the " $\times 2$ map" $T(x)=2 x(\bmod 1)$ provides a favourite example of a dynamical system exhibiting a variety of complicated behaviour. The richness of the dynamics of $T$ is reflected by the diversity of its invariant sets, and more generally of its invariant (Borel) probability measures. Much is known about the set of $\times 2$ invariant measures, though open problems remain, such as the determination of those members which are also $\times 3$-invariant, see e.g. $[13,18,32]$.

Here we shall be interested in the relative dispersion of $\times 2$-invariant measures, declaring one measure to be more diffuse than another if, roughly speaking, its mass lies closer to the boundary of $[0,1)$. With this in mind, it is convenient to extend $T$ to the closed interval $X=[0,1]$ by defining $T(1)=1$, thus effecting a (weak*) compactification of the set of invariant measures. The resulting simplex $\mathcal{M}$ of $T$-invariant Borel probability measures on $X$ is naturally identified with the set of shift-invariant Borel probability measures on $\{0,1\}^{\mathbb{N}}$.

An established notion of the relative dispersion of two probability measures $\mu$ and $\nu$ is the following: we say that $\mu$ is majorized $^{1}$ by $\nu$, and write $\mu \prec \nu$, if $\mu(f) \leq \nu(f)$ for every convex function $f: X \rightarrow \mathbb{R}$. Among all (not necessarily invariant) probability measures on $X$, the least diffuse are the Dirac masses: indeed Jensen's inequality implies that the Dirac mass $\delta_{\varrho}$ is majorized by every probability measure $\mu$ whose barycentre $b(\mu):=\int x d \mu(x)$ equals $\varrho$. The most diffuse probability measures are those concentrated on the boundary of $X: \nu_{\varrho}:=(1-\varrho) \delta_{0}+\varrho \delta_{1}$ majorizes every $\mu$ with $b(\mu)=\varrho$.

If, however, we restrict attention to probability measures invariant under some given transformation of $X$, it is usually a non-trivial problem to determine which measures in this subset are the most, or the least, diffuse. In the case of the $\times 2$ map, the most diffuse measures are in fact easily identified: both endpoints 0 and

Received by the editors September 18, 2007.

${ }^{1}$ While its roots lie in economics [12, 25, 29], the mathematical foundations of majorization were laid by Hardy, Littlewood \& Pólya [15], and later developed notably in [3, 9, 10]. Majorization is the basis for numerous inequalities in pure mathematics (see [27]), while in various applications it is regarded as the proper indicator of relative dispersion (see e.g. $[27,31]$ ). 
1 are fixed, so each measure $\nu_{\varrho}$ is invariant, and majorizes all other members of $\mathcal{M}_{\varrho}:=\{\mu \in \mathcal{M}: b(\mu)=\varrho\}^{2}{ }^{2}$ In other words, each ordered set $\left(\mathcal{M}_{\varrho}, \prec\right)$ has a greatest element, namely $\nu_{\varrho}$, and these measures are precisely the maximal elements of $(\mathcal{M}, \prec)$. Determining the minimal elements of $(\mathcal{M}, \prec)$ is more difficult, however, since the Dirac measures $\delta_{\varrho}$ are not invariant (unless $\varrho=0$ or 1 ). In particular it is unclear, a priori, whether or not each $\left(\mathcal{M}_{\varrho}, \prec\right)$ has a least element.

Remarkably, it turns out that each $\left(\mathcal{M}_{\varrho}, \prec\right)$ does have a least element, which moreover can be identified explicitly:

Theorem 1. For every $\varrho \in[0,1]$, the ordered set $\left(\mathcal{M}_{\varrho}, \prec\right)$ has a least element. This least element is the Sturmian measure $S_{\varrho}$ of rotation number $\varrho$.

The Sturmian measure of rotation number $\varrho$ is defined in terms of a standard symbolic coding procedure induced by the circle rotation of angle $\varrho$. Specifically, for $\varrho \in(0,1)$ it is the push forward of Lebesgue measure on $X$ under the map $x \mapsto$ $\sum_{n>0} \chi_{[1-\varrho, 1)}(\{x+n \varrho\}) / 2^{n+1}$, where $\{\cdot\}$ denotes reduction modulo 1 , and for $\varrho=0$ or 1 it is the Dirac measure supported on the corresponding fixed point. For rational $\varrho$ the Sturmian measure is supported on a single periodic orbit, while for irrational $\varrho$ its support is a uniquely ergodic Cantor set. For example the Sturmian measures of rotation numbers $1 / 2,1 / 3,2 / 5,3 / 8$ and $5 / 13$ are, respectively, the periodic orbit measures corresponding to the strings

$$
\text { 01, 001, 00101, 00100101, 0010010100101, }
$$

while the Sturmian measure of rotation number $(3-\sqrt{5}) / 2$ is the unique invariant probability measure supported by the shift orbit closure of

$$
0010010100100101001010010010100101 \ldots
$$

The terminology Sturmian follows Morse \& Hedlund [28], who coined the term to describe (symbolically) the points in the support of Sturmian measures, so-called Sturmian sequences. A Sturmian sequence of rotation number $\varrho$ is a sequence on the alphabet $\{0,1\}$ in which the symbol 1 occurs with frequency $\varrho$, and as "regularly" as possible ${ }^{3}$. For various other characterizations see e.g. [1, 4, 8, 20, 26, 28, 30].

A result of Cartier [9] asserts that $\mu \prec \nu$ if and only if $\nu$ is a dilation of $\mu$, i.e. there exists a family of probability measures $\left(D_{x}\right)_{x \in X}$, with each $b\left(D_{x}\right)=x$, such that if $f: X \rightarrow \mathbb{R}$ is bounded and Borel then so is $x \mapsto D_{x}(f)$, and $\nu(f)=\int D_{x}(f) d \mu(x)$. Combining this characterization with Theorem 1 gives:

Corollary 1. If $f: X \rightarrow \mathbb{R}$ is strictly convex then for every $\varrho \in[0,1]$, the Sturmian measure $S_{\varrho}$ has strictly smaller $f$-integral than any other measure in $\mathcal{M}_{\varrho}$.

The proof follows from the fact that Jensen's inequality is strict, i.e. $D_{x}(f)>f(x)$, whenever $f$ is strictly convex and $D_{x}$ is not the Dirac measure at $x$. In particular the variance $\operatorname{var}(\mu)=\int(x-b(\mu))^{2} d \mu(x)$ is minimized precisely when $\mu$ is Sturmian:

\footnotetext{
${ }^{2}$ An equivalent definition of $\mathcal{M}_{\varrho}$ is as the set of invariant measures giving weight $\varrho$ to the symbol "1" (i.e. to the half-interval $[1 / 2,1]$ ), since the identity on $X$ differs from the indicator function $\chi_{[1 / 2,1]}$ by $T-$ id, which has zero mean for each invariant measure.

${ }^{3}$ Morse \& Hedlund used the term mechanical to reflect the regularity of such sequences. Many authors prefer the term balanced, reserving the terminology Sturmian for the case of irrational $\varrho$ (see e.g. $[1,26,30])$.
} 
Corollary 2. For every $\varrho \in[0,1]$, the Sturmian measure of rotation number $\varrho$ is the unique measure with smallest variance in $\mathcal{M}_{\varrho}$.

Another consequence is that, among all periodic orbits with a given arithmetic mean, the Sturmian orbit has largest geometric mean:

Corollary 3. Let $x_{1}, \ldots, x_{Q}$ be any non-Sturmian periodic orbit. If $s_{1}, \ldots, s_{q}$ denotes the Sturmian orbit of rotation number $p / q=Q^{-1} \sum_{i=1}^{Q} x_{i}$, then $\left(\prod_{i=1}^{q} s_{i}\right)^{1 / q}>$ $\left(\prod_{i=1}^{Q} x_{i}\right)^{1 / Q}$.

The original motivation for Theorem 1 was its application to ergodic optimization (see e.g. $[5,6,11,21]$ ): for a bounded Borel function $f: X \rightarrow \mathbb{R}$, a measure $\mu \in \mathcal{M}$ is said to be minimizing if $\mu(f)=\inf _{m \in \mathcal{M}} m(f)$ and maximizing if $\mu(f)=\sup _{m \in \mathcal{M}} m(f)$. Since $\varrho \mapsto S_{\varrho}(f)=\inf _{\mu \in \mathcal{M}_{\varrho}} \mu(f)$ is convex, and strictly so when $f$ is strictly convex (a simple consequence of Corollary 1 ), we deduce ${ }^{4}$ :

Corollary 4. If $f: X \rightarrow \mathbb{R}$ is convex then it has a Sturmian minimizing measure. If it is strictly convex then it has a unique minimizing measure, and this measure is Sturmian.

The appearance of Sturmian measures as minimizing and maximizing measures for natural classes of functions is not altogether new: Bousch [4] has shown that every degree-one trigonometric polynomial has unique minimizing and maximizing measures, both of which are Sturmian (this corresponds to the fact that Sturmian measures describe the boundary of the "poisson" $\left.\left\{\int \exp (2 \pi i t) d \mu(t): \mu \in \mathcal{M}\right\} \subset \mathbb{C}\right)$. A significant difference is that, for convex functions, the Sturmian nature of the minimizing measure is a consequence of solving a family of restricted variational problems. For degree-one trigonometric polynomials $f$ there is no analogue of Theorem 1: it is not the case that every Sturmian measure has smaller $f$-integral than all other invariant measures of same barycentre.

Investigation of the ordered set $(\mathcal{M}, \prec)$ is facilitated by the fact that certain $\times 2$ invariant measures are known "explicitly" (Lebesgue measure, periodic orbit measures), while others can be well-approximated, either by periodic orbit measures or via explicit symbolic coding. For example, part of $\left(\mathcal{M}_{1 / 2}, \prec\right)$ is depicted in the Hasse diagram $^{5}$ of Figure 1; here each binary string represents the invariant probability measure supported by the corresponding periodic orbit, so for example Morse measure (concentrated on the shift orbit closure of the Morse sequence $0110100110010110 \ldots$. ) majorizes $01=\{1 / 3,2 / 3\}$, is majorized by $000111=\{1 / 9, \ldots, 8 / 9\}$, and is incomparable with $0011=\{1 / 5,2 / 5,3 / 5,4 / 5\}$.

To derive the above relations, and more generally to decide if and how two measures in $\mathcal{M}$ are related, one may exploit the characterisation (see $[23,24,31]$ ) that $\mu \prec \nu$ if and only if $b(\mu)=b(\nu)$ and $\int_{0}^{t} \mu[0, x] d x \leq \int_{0}^{t} \nu[0, x] d x$ for all $t \in[0,1]$. In the case where $\mu$ and $\nu$ are purely atomic and each atom has equal weight (e.g. if they are periodic orbit measures of the same period), this recovers the famous HardyLittlewood-Pólya criterion for majorization (see $[15,16]$ ): if $b(\mu)=b(\nu)$ then $\mu \prec \nu$

\footnotetext{
${ }^{4}$ Note that determining the maximizing measure for a convex function is trivial, because the endpoints of $X$ are both fixed by $T$.

${ }^{5}$ See $[22]$ for a Hasse diagram of a larger portion of $\left(\mathcal{M}_{1 / 2}, \prec\right)$.
} 


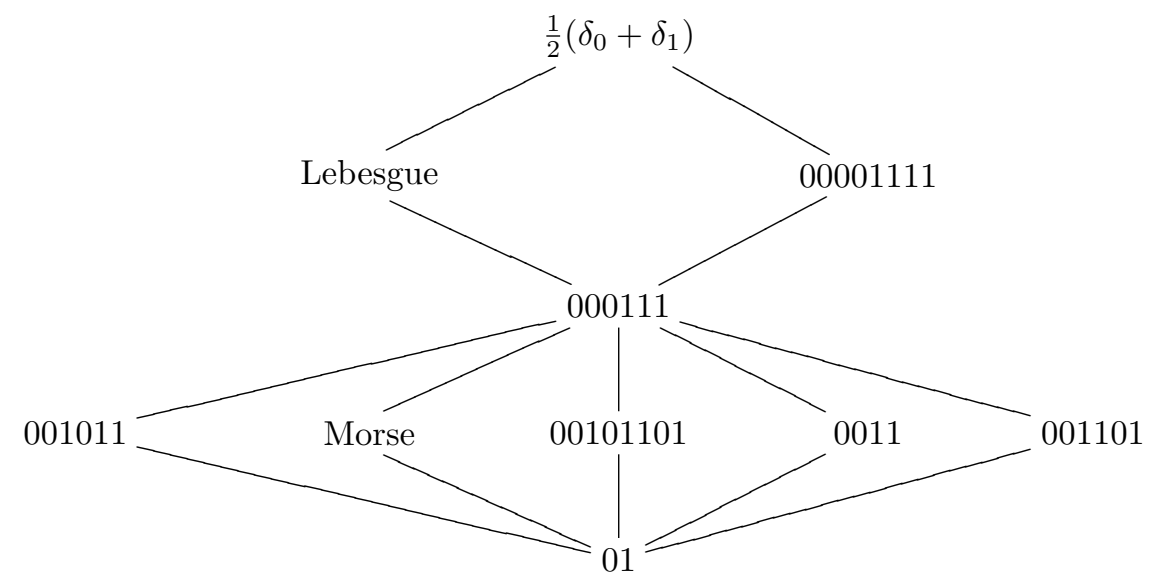

Figure 1. Hasse diagram of part of $\mathcal{M}_{1 / 2}$

if and only if $\sum_{i=1}^{n} \mu_{i} \geq \sum_{i=1}^{n} \nu_{i}$ for all $1 \leq n \leq Q-1$, where $\mu:=Q^{-1} \sum_{i=1}^{Q} \delta_{\mu_{i}}$, $\nu:=Q^{-1} \sum_{i=1}^{Q} \delta_{\nu_{i}}$, with $\mu_{1} \leq \ldots \leq \mu_{Q}$ and $\nu_{1} \leq \ldots \leq \nu_{Q}$. For example, combining this criterion with Theorem 1 yields:

Corollary 5. Let $\mu_{1}<\ldots<\mu_{Q}$ be any periodic orbit, and $p / q=Q^{-1} \sum_{i=1}^{Q} \mu_{i}$ its arithmetic mean, where $1 \leq p<q$ are coprime integers. If $s_{1}<\ldots<s_{q}$ are the points in the Sturmian orbit of rotation number $p / q$, and $s_{i+(j-1) q}^{\prime}:=s_{j}$ for $1 \leq i \leq q$, $1 \leq j \leq Q / q$, then

$$
\sum_{i=1}^{n} s_{i}^{\prime} \geq \sum_{i=1}^{n} \mu_{i} \quad \text { for all } \quad 1 \leq n \leq Q-1
$$

If the orbit $\mu_{1}<\ldots<\mu_{Q}$ is non-Sturmian then the extreme cases $n=1$ and $n=Q-1$ of (1) clearly become strict inequalities, $s_{1}>\mu_{1}$ and $s_{q}<\mu_{Q}$; these two inequalities were first proved, using different methods, by Bernhardt [2] (see also [14]). In fact a modification of the proof below yields a stronger result, namely that $s_{1}$ is strictly greater than $\min (\operatorname{supp}(\mu))$, and $s_{q}$ is strictly smaller than $\max (\operatorname{supp}(\mu))$, for any non-Sturmian measure $\mu \in \mathcal{M}_{p / q}$.

Notation. The Lebesgue measure of a set $E$ is denoted by $|E|$, and the Lebesgue integral of a function $g$ by $\int g$. For other measures $\mu$ we write either $\mu(g)$ or $\int g d \mu$ for the integral of $g$.

\section{Proof of Theorem 1}

If $\varrho$ equals 0 or 1 then $\mathcal{M}_{\varrho}$ is a singleton, and Theorem 1 is trivially true, so suppose $\varrho \in(0,1)$. Since $C^{2}$ convex functions are weakly dense among all convex functions, it suffices to show that if $f$ is $C^{2}$ convex then $S_{\varrho}(f) \leq \mu(f)$ for all $\mu \in \mathcal{M}_{\varrho}$. If $f_{\theta}: X \rightarrow \mathbb{R}$ is defined, for $\theta \in \mathbb{R}$, by $f_{\theta}(x)=f(x)+\theta x$, this is equivalent to

$$
S_{\varrho}\left(f_{\theta}\right) \leq \mu\left(f_{\theta}\right) \text { for all } \mu \in \mathcal{M}_{\varrho} .
$$


The strategy, therefore, will be to find, for each $\varrho \in(0,1)$, a value $\theta \in \mathbb{R}$ for which (2) holds. In fact $\theta$ can be chosen with the stronger property that $S_{\varrho}$ is a minimizing measure for $f_{\theta}$, a result described by the following Theorem 2, which in particular implies Theorem 1.

Theorem 2. Let $f: X \rightarrow \mathbb{R}$ be a $C^{2}$ convex function. For every $\varrho \in(0,1)$ there exists $\theta \in \mathbb{R}$ such that the Sturmian measure $S_{\varrho}$ is a minimizing measure for the function $f_{\theta}$ defined by $f_{\theta}(x)=f(x)+\theta x$.

Proof. There exists $\gamma \in(0,1 / 2)$ such that the support of $S_{\varrho}$ is contained in $H_{\gamma}=$ $[\gamma, \gamma+1 / 2]$ (see e.g. $[7,8,14,34]){ }^{6}$ Define $\tau=\tau_{\gamma}: X \rightarrow X$ by

$$
\tau_{\gamma}(x)= \begin{cases}(x+1) / 2 & \text { if } x \in[0,2 \gamma) \\ x / 2 & \text { if } x \in[2 \gamma, 1]\end{cases}
$$

If $\theta:=-\int \sum_{n=1}^{\infty} 2^{-n} f^{\prime} \circ \tau^{n}$ then the $L^{\infty}$ function $\sum_{n=1}^{\infty} 2^{-n} f_{\theta}^{\prime} \circ \tau^{n}$ has Lebesgue integral zero, so is the almost everywhere derivative of a Lipschitz function $\varphi_{\theta}: X \rightarrow \mathbb{R}$ satisfying $\varphi_{\theta}(0)=\varphi_{\theta}(1)$. Now $\left(f_{\theta}+\varphi_{\theta}-\varphi_{\theta} \circ T\right)^{\prime}=0$ Lebesgue almost everywhere on $H_{\gamma}$, because $\tau \circ T$ is, Lebesgue almost everywhere, the identity function on $H_{\gamma}$. Therefore, since $f_{\theta}+\varphi_{\theta}-\varphi_{\theta} \circ T$ is absolutely continuous on $X$, its restriction to $H_{\gamma}$ is constant. We claim that the constant value taken by $f_{\theta}+\varphi_{\theta}-\varphi_{\theta} \circ T$ on $H_{\gamma}$ is in fact its minimum, i.e. that

$$
\left(f_{\theta}+\varphi_{\theta}\right)(s) \leq \begin{cases}\left(f_{\theta}+\varphi_{\theta}\right)(s+1 / 2) & \text { for } s \in(\gamma, 1 / 2] \\ \left(f_{\theta}+\varphi_{\theta}\right)(s-1 / 2) & \text { for } s \in(1 / 2, \gamma+1 / 2)\end{cases}
$$

From this it follows that $S_{\varrho}$ is a minimizing measure for $f_{\theta}+\varphi_{\theta}-\varphi_{\theta} \circ T$, and hence for $f_{\theta}$.

To prove the first inequality in $(3)$ let $s \in(\gamma, 1 / 2]$, so that

$$
\begin{aligned}
\left(f_{\theta}+\varphi_{\theta}\right)(s)-\left(f_{\theta}+\varphi_{\theta}\right)(s+1 / 2) & =\int_{\gamma}^{s}\left(f_{\theta}+\varphi_{\theta}\right)^{\prime}-\int_{\gamma+1 / 2}^{s+1 / 2}\left(f_{\theta}+\varphi_{\theta}\right)^{\prime} \\
& =\sum_{n=0}^{\infty}\left[\int_{E} 2^{-n} f_{\theta}^{\prime} \circ \tau^{n}-\int_{E^{\prime}} 2^{-n} f_{\theta}^{\prime} \circ \tau^{n}\right] \\
& =\sum_{n=0}^{\infty}\left[\int_{\tau^{n}(E)} f_{\theta}^{\prime}-\int_{\tau^{n}\left(E^{\prime}\right)} f_{\theta}^{\prime}\right] \\
& =\int C_{s} \cdot f_{\theta}^{\prime},
\end{aligned}
$$

where

$$
C_{s}:=\sum_{n=0}^{\infty}\left[\chi\left(\tau^{n} E\right)-\chi\left(\tau^{n} E^{\prime}\right)\right], E=E_{s}:=(\gamma, s], E^{\prime}=E_{s}^{\prime}:=(\gamma+1 / 2, s+1 / 2] .
$$

Now $C_{s}(0)=0$, and $C_{s}$ is Lebesgue-integrable, with $\int C_{s}=0$, because $\left|\tau^{n} E\right|=$ $2^{-n}|E|=\left|\tau^{n} E^{\prime}\right|$ for all $n \geq 0$. If $B_{s}(t):=\int_{0}^{t} C_{s}$ then $B_{s}(0)=0=B_{s}(1)$, so

\footnotetext{
${ }^{6}$ The value $\gamma$ is unique if and only if $\varrho$ is irrational. $S_{\varrho}$ is the unique invariant measure for the continuous degree-one map of the circle $\left([0,1]\right.$ with endpoints identified) whose restriction to $H_{\gamma}$ is $T$ and which is constant on the complement of $H_{\gamma}$.
} 
integration by parts yields $\left(f_{\theta}+\varphi_{\theta}\right)(s)-\left(f_{\theta}+\varphi_{\theta}\right)(s+1 / 2)=-\int B_{s} \cdot f_{\theta}^{\prime \prime}$. Now $f$ is convex, therefore $f_{\theta}^{\prime \prime}=f^{\prime \prime} \geq 0$, so the required inequality will follow if it can be shown that $B_{s}$ is non-negative on $X$.

For this, first note that $C_{s}$ is identically zero on $[0, \gamma]$, hence so is $B_{s}$. Since $E^{\prime}$ and $H_{\gamma}$ are disjoint, $\tau^{m}\left(E^{\prime}\right) \cap \tau^{n}\left(E^{\prime}\right)=\emptyset$ for $m \neq n$, so $\sum_{n=0}^{\infty} \chi\left(\tau^{n}\left(E^{\prime}\right)\right) \leq 1$. In particular, $C_{s} \geq 1-\sum_{n=0}^{\infty} \chi\left(\tau^{n}\left(E^{\prime}\right)\right) \geq 0$ on $E=(\gamma, s]$, hence $B_{s} \geq 0$ on $(\gamma, s]$ as well. So $B_{s}(t) \geq 0$ for $t \in[0, s]$. Now $C_{s}$ is identically zero on $(s+1 / 2,1]$, and equal to -1 on $E^{\prime}=(\gamma+1 / 2, s+1 / 2]$, so $B_{s}(t)=\int_{0}^{t} C_{s}=-\int_{t}^{1} C_{s}=s+1 / 2-t>0$ for $t \in[\gamma+1 / 2, s+1 / 2)$, and $B_{s}(t)=0$ for $t \in[s+1 / 2,1]$. If $t \in(s, \gamma+1 / 2)$ then

$$
\begin{aligned}
B_{s}(t) & =\int_{0}^{t} C_{s}=\int_{\gamma}^{t} C_{s} \\
& =\sum_{n=0}^{\infty} \int_{\gamma}^{t} \chi\left(\tau^{n}(E)\right)-\chi\left(\tau^{n}\left(E^{\prime}\right)\right) \\
& \geq|(\gamma, t] \cap E|-\left|(\gamma, t] \cap E^{\prime}\right|-\sum_{n=1}^{\infty}\left|(\gamma, t] \cap \tau^{n} E^{\prime}\right| \\
& \geq|E|-\sum_{n=1}^{\infty}\left|\tau^{n} E^{\prime}\right|=|E|\left(1-\sum_{n=1}^{\infty} 2^{-n}\right)=0 .
\end{aligned}
$$

The second inequality in (3) is proved similarly: if $s \in(1 / 2, \gamma+1 / 2)$ then

$$
\left(f_{\theta}+\varphi_{\theta}\right)(s)-\left(f_{\theta}+\varphi_{\theta}\right)(s-1 / 2)=\int \tilde{C}_{s} \cdot f_{\theta}^{\prime}=-\int \tilde{B}_{s} \cdot f^{\prime \prime},
$$

where $\tilde{C}_{s}:=\sum_{n=0}^{\infty} \chi\left(\tau^{n}\left(D^{\prime}\right)\right)-\chi\left(\tau^{n}(D)\right), D^{\prime}=[s-1 / 2, \gamma), D=[s, \gamma+1 / 2)$, $\tilde{B}_{s}(t):=\int_{0}^{t} \tilde{C}_{s}$, and an argument analogous to the one above can be used to show that $\tilde{B}_{s}$ is non-negative on $X$.

\section{Remark 1.}

(a) The choice of $\theta$ in Theorem 2 is inspired by Bousch's précondition de Sturm [4], cf. [17].

(b) If $f_{\theta}+\varphi_{\theta}$ happens to itself be convex (in general it is not, despite its second derivative being Lebesgue almost everywhere positive), the key inequality (3) is immediate.

\section{Acknowledgments}

This project began in 1997, from work [19, 20] towards the conjecture that Sturmian measures describe the boundary of the "poisson", a result discovered independently and subsequently proved by Thierry Bousch [4]. It gained impetus when Peter Gibson and Mike Taylor informed me of the work of Hardy, Littlewood \& Pólya relating doubly stochastic matrices to majorization. I am grateful to Thierry Bousch for encouraging me to pursue the project, and for sharing his ideas on how to resolve it; in particular, the proof given here follows a suggestion of Thierry. Since 2003 I have benefited from EPSRC support in the form of an Advanced Research Fellowship. 


\section{References}

[1] J.-P. Allouche \& J. Shallit, Automatic sequences. Theory, applications, generalizations, Cambridge University Press, Cambridge, 2003.

[2] C. Bernhardt, A class of endomorphisms of the circle, Proc. London Math. Soc., 45 (1982), $258-280$.

[3] D. Blackwell, Equivalent comparisons of experiments, Ann. Math. Statist. 24 (1953), 265-272.

[4] T. Bousch, Le poisson n'a pas d'arêtes, Ann. Inst. Henri Poincaré (Proba. et Stat.) 36, (2000), 489-508.

[5] _ La condition de Walters, Ann. Sci. ENS, 34, (2001), 287-311.

[6] T. Bousch \& O. Jenkinson, Cohomology classes of dynamically non-negative $C^{k}$ functions, Invent. Math. 148 (2002), 207-217.

[7] T. Bousch \& J. Mairesse, Asymptotic height optimization for topical IFS, tetris heaps, and the finiteness conjecture, Jour. Amer. Math. Soc. 15 (2002), 77-111.

[8] S. Bullett \& P. Sentenac, Ordered orbits of the shift, square roots, and the devil's staircase, Math. Proc. Camb. Phil. Soc. 115 (1994), 451-481.

[9] P. Cartier, J. M. G. Fell, \& P.-A. Meyer, Comparaison des mesures portées par un ensemble convexe compact, Bull. Soc. Math. France, 92 (1964), 435-445.

[10] G. Choquet, Le théorème de représentation intégrale dans les ensembles convexes compacts, Ann. Inst. Fourier (Grenoble), 10 (1960), 333-344.

[11] G. Contreras, A. Lopes, \& Ph. Thieullen, Lyapunov minimizing measures for expanding maps of the circle, Ergod. Th. \& Dyn. Sys. 21 (2001), 1379-1409.

[12] H. Dalton, The measurement of the inequality of incomes, Econom. J. 30 (1920), 348-361.

[13] H. Furstenberg, Disjointness in ergodic theory, minimal sets, and a problem in Diophantine approximation, Math. Systems Theory, 1 (1967), 1-49.

[14] J-M. Gambaudo, O. Lanford III, \& C. Tresser, Dynamique symbolique des rotations, C. R. Acad. Sc. Paris, 299 (1984), 823-826.

[15] G. H. Hardy, J. E. Littlewood, \& G. Pólya, Some simple inequalities satisfied by convex functions, Messenger Math. 58 (1929), 145-152.

[16] _ Inequalities, Cambridge University Press, Cambridge, (1st ed., 1934; 2nd ed., 1952).

[17] E. Harriss \& O. Jenkinson, Flattening functions on flowers, Ergod. Th. \& Dyn. Sys. 27 (2007), $1865-1886$.

[18] B. Host, Nombres normaux, entropie, translations, Israel J. Math. 91 (1995), 419-428.

[19] O. Jenkinson, Conjugacy rigidity, cohomological triviality, and barycentres of invariant measures, Ph. D. thesis, University of Warwick, 1996.

[20] _ Frequency locking on the boundary of the barycentre set, Experimental Mathematics, 9 (2000), 309-317.

[21] _ Ergodic optimization, Discrete \& Cont. Dyn. Sys. 15 (2006), 197-224.

[22] _ Optimization and majorization of invariant measures, Electron. Res. Announc. Amer. Math. Soc. 13 (2007), 1-12.

[23] J. Karamata, Sur une inégalité rélative aux fonctions convexes, Publ. Math. Univ. Belgrade, 1 (1932), 145-148.

[24] S. Karlin \& A. Novikoff, Generalized convex inequalities, Pacific J. Math. 13 (1963), 1251-1279.

[25] M. O. Lorenz, Methods of measuring concentration of wealth, J. Amer. Statist. Assoc. 9 (1905), 209-219.

[26] M. Lothaire, Algebraic combinatorics on words, Encyclopaedia of mathematics and its applications, vol. 90, Cambridge University Press, 2002.

[27] A. W. Marshall \& I. Olkin, Inequalities: theory of majorization and its applications, Mathematics in science and engineering vol. 143, Academic Press, 1979.

[28] M. Morse \& G. A. Hedlund, Symbolic Dynamics II. Sturmian Trajectories, Amer. J. Math. 62 (1940), 1-42.

[29] A. C. Pigou, Wealth and welfare, Macmillan, New York, 1912.

[30] N. Pytheas Fogg, Substitutions in dynamics, arithmetics and combinatorics, Springer Lecture Notes in Mathematics vol. 1794, 2002.

[31] M. Rothschild \& J. Stiglitz, Increasing risk I: A definition, J. of Economic Theory, 2 (1970), $225-243$. 
[32] D. Rudolph, $\times 2$ and $\times 3$ invariant measures and entropy, Ergod. Th. \& Dyn. Sys. 10 (1990), 395-406.

[33] I. Schur, Über eine Klasse von Mittelbildungen mit Anwendungen die Determinanten, Theorie Sitzungsber. Berlin. Math. Gesellschaft, 22 (1923), 9-20 (Issai Schur colleted works, (A. Brauer \& H. Rohrbach, eds.) Vol. II, pp. 416-427, Springer-Verlag, Berlin, 1973).

[34] P. Veerman, Symbolic dynamics of order-preserving orbits, Physica D, 29 (1987), 191-201.

Oliver Jenkinson; School of Mathematical Sciences, Queen Mary, University of LonDon, Mile End Road, London, E1 4NS, UK.

E-mail address: omj@maths.qmul.ac.uk

$U R L$ : www . maths.qmul.ac.uk/ omj 